EPJ Web of Conferences 47, 03008 (2013)

DOI: $10.1051 /$ epjconf/20134703008

(C) Owned by the authors, published by EDP Sciences, 2013

\title{
MASCARA: The Multi-site All-Sky CAmeRA
}

\author{
Inas Snellen ${ }^{1, a}$, Remko Stuik ${ }^{1}$, Gilles Otten ${ }^{1}$, Felix Bettonvil ${ }^{2}$, \\ Ramon Navarro ${ }^{2}$, Matthew Kenworthy ${ }^{1}$, Ernst de Mooij ${ }^{3}$, Rik ter Horst ${ }^{2}$, \\ Rudolf Le Poole ${ }^{1}$, Anna-Lea Lesage ${ }^{1}$ and Julien Spronck ${ }^{1}$ \\ ${ }^{1}$ Leiden Observatory, Leiden University, Postbus 5913, 2300 RA, Leiden, The Netherlands \\ ${ }^{2}$ NOVA Optical and Infrared Instrumentation Division at ASTRON, PO Box 2, 7990 AA, \\ Dwingeloo, The Netherlands \\ ${ }^{3}$ Department of Astronomy and Astrophysics, University of Toronto, 50 St. George Street, \\ Toronto, ON M5S 3H4, Canada
}

\begin{abstract}
The Multi-site All-Sky CAmeRA, MASCARA, is an instrument currently in the design phase, that is aimed to find the brightest transiting planet systems in the sky. It will consist of several stations across the globe, each monitoring the near-entire sky using a battery of CCD-detectors plus wide-field lenses, targeting stars in the $\mathrm{V}=4-8$ magnitude range. MASCARA will be able to detect individual transits from Jupiter-size planets over this whole magnitude range, while smaller planets will be found by co-adding transit events. We expect to discover up to a dozen bright transit systems in this way. These will be extremely valuable for atmospheric follow-up studies.
\end{abstract}

\section{BRIGHT TRANSITING PLANET SYSTEMS}

Ground-based and space-based transit surveys, such as HATNet [1], SuperWASP [2], CoRoT [3], and Kepler [4], have been extremely successful in finding transiting exoplanets. However, they have not been designed to find the brightest and most nearby transiting systems. The current ground-based surveys are limited to stars with $\mathrm{V}>8$ due to saturation issues. The Kepler and CoRoT satellites survey only relatively small areas of sky, meaning that they generally discover planets transiting much fainter stars (see Fig. 1). Bright transiting planet systems are crucial for follow-up studies. The most detailed atmospheric characterization of exoplanets has been conducted on two planets, HD189733b and HD209458b, both of $\mathrm{V}=7.7$ and first discovered via radial velocity $[5,6]$ after which they were found to transit. Atmospheric characterization through transmission spectroscopy and secondary eclipse spectral-photometry of these systems, both from space and the ground, has led to the identification of several molecular and atomic gases, and to constraints on their atmospheric temperature structures and global circulation [7-13].

Current-day instrumentation is simply not sensitive enough to detect such atmospheric features, present at a $\sim 10^{-3-4}$ photometric level, in significantly fainter targets. Furthermore, when the nexgeneration space-based and ground-based instrumentation comes online, such as the James Webb Telescope (JWST), the Exoplanet Characterization Observatory (EChO), and the Extremely Large Telescopes (ELTs), transiting systems of similar apparent magnitudes are required to extend these observations to smaller and cooler planets. Although a few transiting systems, such as HD189733 and HD209458 have been discovered via radial velocity, only a fraction of the stars in the

\footnotetext{
ae-mail: snellen@strw.leidenuniv.nl
}

This is an Open Access article distributed under the terms of the Creative Commons Attribution License 2.0, which permits unrestricted use, distribution, and reproduction in any medium, provided the original work is properly cited. 


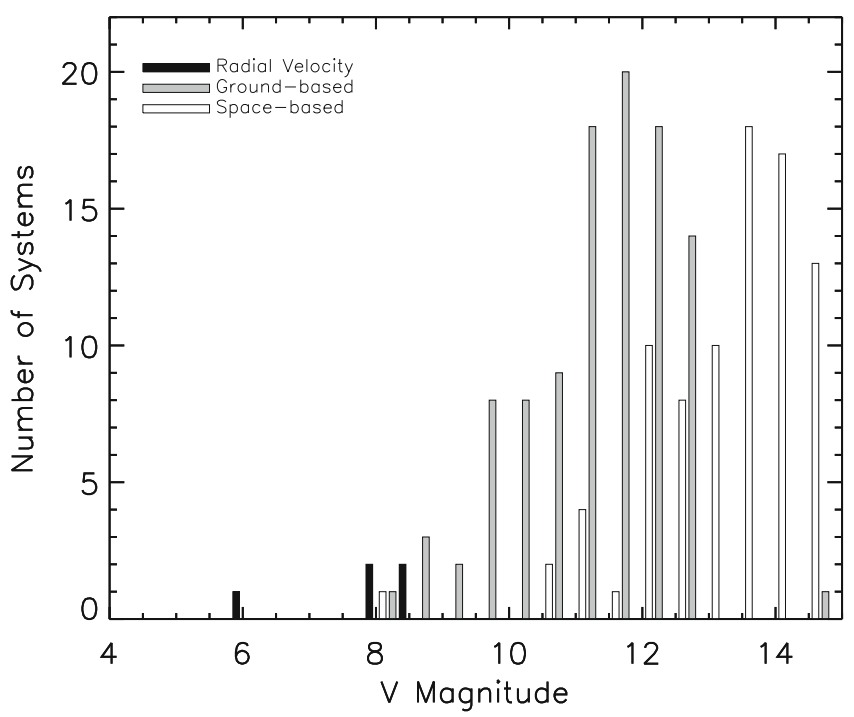

Figure 1. Number of discoveries of transiting extrasolar planets as function of apparent V magnitude, showing those systems discovered first by radial velocity (dark histograms), and by ground-based or space based surveys (grey and light histograms). The data are taken from exoplanet.eu (December 2012).

$\mathrm{V}=4-8$ magnitude range have been RV monitored, and most of them only at a level such that Jupitermass planets would have been detected. For example, currently the HARPS team monitors only ten of the brightest and quietest solar-type stars to find Earth-mass planets in their habitable zones [14]. This means that an unbiased photometric survey such as MASCARA is needed to find the brightest transiting systems.

Both the radial velocity surveys and the Kepler and CoRoT transit surveys teach us that there should exist a rich and versatile population of transiting planets at bright optical magnitudes. There are 800010000 main sequence stars at $\mathrm{V}<8$, of which the majority are of $\mathrm{A}$ and $\mathrm{F}$ stellar type. Assuming a $1 \%$ incidence of hot Jupiters with a transit probability of $10 \%$, this implies that more than a handful of transiting hot Jupiters are still to be discovered at these bright magnitudes. The steep mass-function of extrasolar planets means that smaller transiting planets will be present at even brighter magnitudes. Indeed, $55 \mathrm{Cnc}$ e is the first known planet to transit a naked eye star $(\mathrm{V}=5.95)[15,16]$. Several of these objects should exist at similar magnitudes. Within a few years, MASCARA observations will have covered sufficient transits of these targets to allow secure detections.

\section{THE MASCARA CONCEPT AND ROLL-OUT}

The MASCARA camera system will have no moving parts [17]. Due to the near all-sky nature of the observations, there is no need for tracking to compensate for the Earth rotation. In the current baseline design, a MASCARA station consists of four Atik $11000 \mathrm{CCD}$ cameras, each with $4 \mathrm{k} \times 2.67 \mathrm{k} 9 \mu \mathrm{m}$ pixels, and equipped with a $20 \mathrm{~mm} \mathrm{F/2.8} \mathrm{wide-field} \mathrm{Canon} \mathrm{lens.} \mathrm{The} \mathrm{cameras} \mathrm{wil} \mathrm{be} \mathrm{pointed} \mathrm{in} \mathrm{such} \mathrm{way}$ that at each site most of the sky at airmass $<3$ will be covered. Exposure times will be kept short to 3-6 seconds (matching the total read-out time of the interline CCD arrays), to avoid trailing of the stars on the CCD. The use of short exposures implies that the data rate will reach 1 tera-byte per night per station, meaning that a significant part of the data reduction will need to be done on site in real time. Although a data buffer worth one week of observations will be stored locally, which can be retrieved in the case of important transient events, in general the raw data will not be stored permanently - just like in the case of the space-based transit surveys. 


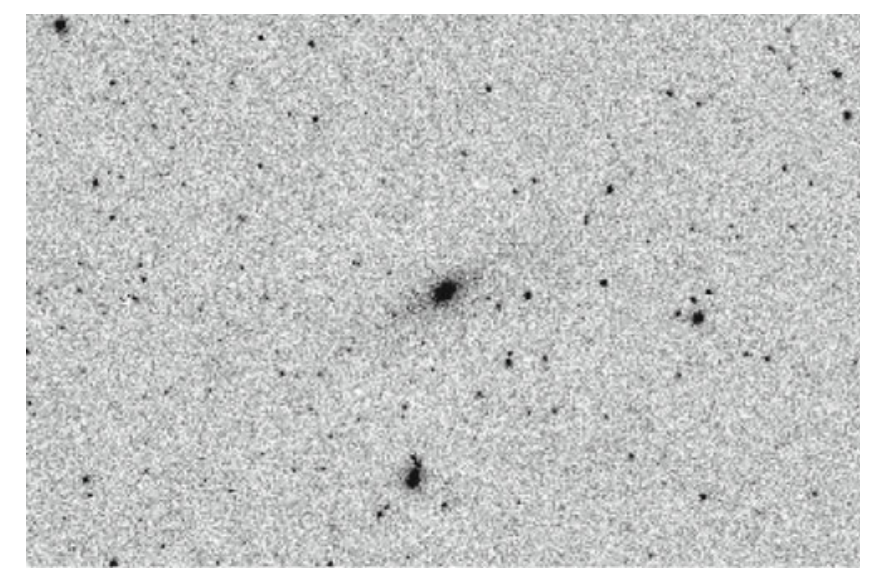

Figure 2. A 3-sec test observation taken with the MASCARA camera set-up from Leiden in the Netherlands, showing $\sim 1 / 150$ th of the full frame centered on M31. Stars down to $V=9$ are clearly visible.

We recently performed our first on-sky tests of the Atik 11000 CCD camera. Figure 2 shows a 3 sec exposure taken from Leiden in the Netherlands. Stars down to $\mathrm{V}=9$ are clearly detected. This means that we expect to surpass our goal of $1 \%$ photometry per hour for $\mathrm{V}=8$ stars [17] by a factor of a few. The first half of 2013 will be used to finalize the MASCARA design and build the first station in the Netherlands. After testing we aim to install this first station on the Canary Islands by the end of 2013. We expect to roll out the remaining stations, which will be distributed in longitude across both the northern and southern hemispheres [17] in 2014-1015.

\section{References}

[1] Bakos G., JAVSO 40, 241 (2012)

[2] Pollacco D.L. et al. PASP 118, 1407 (2006)

[3] Leger A. et al. A\&A 506, 287 (2009)

[4] Borucki W.J. et al., Science 327, 997 (2010)

[5] Charbonneau D., Brown T.M., Latham D.W., Mayor M., ApJ 529, 45 (2000)

[6] Bouchy F. et al. A\&A 444, 15 (2005)

[7] Knutson H.A. et al. Nature 447, 183 (2007)

[8] Grillmair C.J. et al. Nature 456, 767 (2008)

[9] Charbonneau D., Brown T., Noyes R.W., Gilliland R.L. ApJ 568, 377 (2002)

[10] Beaulilieu J.P. et al. MNRAS 409, 963 (2010)

[11] Snellen I.A.G., Albrecht S., de Mooij E.J.W., Le Poole R.S. A\&A 487, 357 (2008)

[12] Snellen I.A.G., de Kok R.J., de Mooij E.J.W., Albrecht S., Nature 465, 1049 (2010)

[13] Swain M. et al. ApJ 704, 1616 (2009)

[14] Pepe F. et al. A\&A 534, A58 (2011)

[15] Demory B.-O. et al. A\&A 533, A114 (2011)

[16] Winn J. et al., ApJ 737, L18 (2011)

[17] I.A.G. Snellen et al., SPIE 8444, OI (2012) 